The Astrophysical Journal, 563:L49-L52, 2001 December 10

(C) 2001. The American Astronomical Society. All rights reserved. Printed in U.S.A.

\title{
A POSSIBLE FAINT NEAR-INFRARED COUNTERPART TO THE ANOMALOUS X-RAY PULSAR 1E $2259+586$
}

\author{
F. Hulleman, ${ }^{1}$ A. F. Tennant, ${ }^{2}$ M. H. van Kerkwijk, ${ }^{1}$ S. R. Kulkarni, ${ }^{3}$ C. Kouveliotou, ${ }^{4,2}$ And S. K. Patel ${ }^{4}$ \\ Received 2001 August 24; accepted 2001 November 5; published 2001 November 29
}

\begin{abstract}
We present near-infrared and optical observations of the field of the anomalous X-ray pulsar 1 E $2259+586$ taken with the Keck telescope. We derive a subarcsecond Chandra position and tie it to our optical reference frame using other stars in the field. We find a very faint source, $K_{s}=21.7 \pm 0.2$ mag, with a position coincident with the Chandra position. We argue that this is the counterpart. In the $J, I$, and $R$ bands, we derive $(2 \sigma)$ limits of 23.8, 25.6, and $26.4 \mathrm{mag}$, respectively. As with $4 \mathrm{U} 0142+61$, for which a counterpart has previously been found, our results are inconsistent with models in which the source is powered by accretion from a disk but may be consistent with the magnetar model.
\end{abstract}

Subject headings: stars: neutron - X-rays: individual (1E 2259+586) - X-rays: stars

\section{INTRODUCTION}

$1 \mathrm{E} 2259+586$ is a member of a class of X-ray pulsars whose energy source is not understood, called "anomalous X-ray pulsars" (AXPs; see Israel et al. 2002 for a review). Their rotational energy loss is insufficient to explain their X-ray luminosities, and there is no evidence for a companion star from which matter could be accreted.

In part, the uncertainty about AXPs arises because of a lack of counterparts at other wavelengths. Therefore, we have initiated a program of deep optical and near-infrared observations of those AXPs that are not too highly absorbed. This program has led to the detection of the first optical counterpart of an AXP, namely, the counterpart to 4U 0142+61 (Hulleman et al. 2000a). Our earlier observations of 1E $2259+586$ set stringent limits $(R>25.7, I>24.3 \mathrm{mag}$; Hulleman et al. 2000b).

In this Letter, we present in $\S 2$ deeper optical observations and new near-infrared observations of $1 \mathrm{E} 2259+586$. In $\S 3$, we derive an accurate position from Chandra observations (see the companion Letter by Patel et al. 2001), which we use in $\S 4$ to identify a possible near-infrared counterpart to 1E $2259+586$.

\section{OBSERVATIONS}

\subsection{Optical Observations}

Deep $R$ - and $I$-band images of the field around 1E $2259+586$ were taken on the night of 2000 September 3, with the Low Resolution Imager Spectrograph (LRIS; Oke et al. 1995) mounted on Keck I. The night was photometric and the seeing was $0.6\left(0{ }^{\prime \prime} 9\right)$ in $R(I)$. Six (12) images were averaged for a total integration time of 1 hour in $R(I)$. Less deep, wider field of view, $B, V, R$, and $I$ images were taken at the 60 inch $(1.5$ $\mathrm{m})$ telescope on Palomar mountain on the night of 2000 November 18 and were used for the astrometry $(\$ 3)$.

The reduction was done using the ESO-MIDAS software package, in the same way as described by Hulleman et al. (2000b) and Hulleman et al. (2000a). The stacked Keck I-band image is shown in Figure 1. The Keck $R$-band image is not

\footnotetext{
${ }^{1}$ Utrecht University, P.O. Box 80000, 3508 TA Utrecht, Netherlands.

${ }^{2}$ NASA Marshall Space Flight Center, Huntsville, AL 35812.

${ }^{3}$ Palomar Observatory, California Institute of Technology, MS 105-24, Pasadena, CA 91125.

${ }^{4}$ Universities Space Research Association/NSSTC, SD-50, Huntsville, AL 35805 .
}

shown. It is very similar to the image shown in Hulleman et al. (2000b).

We measured instrumental magnitudes on the Keck images using point-spread function (PSF) fitting as implemented in the DAOPHOT package and calibrated these using the calibration found earlier (Hulleman et al. 2000b). We estimate single-trial $2 \sigma$ detection limits of 26.4 (25.6) mag in $R(I)$ by adding 8.1 (7.6) mag to the mean instrumental magnitude of objects that have a PSF fitting error of 0.3 mag. ${ }^{5}$

\subsection{Near-Infrared Photometry}

Near-infrared images in the $J$ and $K_{s}$ filters were obtained on the nights of 1999 June 23 and 24, with the Near Infrared Camera (NIRC; Matthews \& Soifer 1994), mounted on the Keck I telescope. During both nights observing conditions were photometric. During the first night, the seeing was mediocre, varying between 0.6 and 1 .".5, but during the second night it was excellent, at 0.5 in both $K_{s}$ and $J$. Therefore, we use only the data taken on the second night. These consist of a total of 36 frames in each of $J$ and $K_{s}$, with each $K_{s}(J)$ frame composed of five (three) co-added exposures of 12 (20) s.

The data were reduced using MIDAS. After subtracting dark frames, a flat field and a mosaic were created for each filter in the following way. First, each individual frame was normalized to unity. Next, at each pixel the variance over all the (normalized) frames was computed. For a field that is not too crowded, the median of these variances should be a good approximation of the expected variance of a pixel that in each frame contains only sky. Then, for each pixel we used an $F$-test to determine if its variance was significantly higher than the median variance, where we set the level of significance at 0.001 . If the variance was not significantly higher, we simply took the mean of all frames at that pixel. Otherwise, we discarded the highest values at each pixel until the variance was consistent with the median variance. From the values remaining, a new mean was calculated.

After division by this flat field, we used the prescription of Liu \& Graham (2001) to correct each frame for a persistence effect in the readout amplifiers and subtracted the background, which was determined from the frame itself.

A few well-detected stars were used to determine the spatial offsets between individual frames. Next, at each sky position

\footnotetext{
${ }^{5}$ The detailed simulations described in Hulleman et al. (2000b) convinced us that this procedure gives correct estimates.
} 

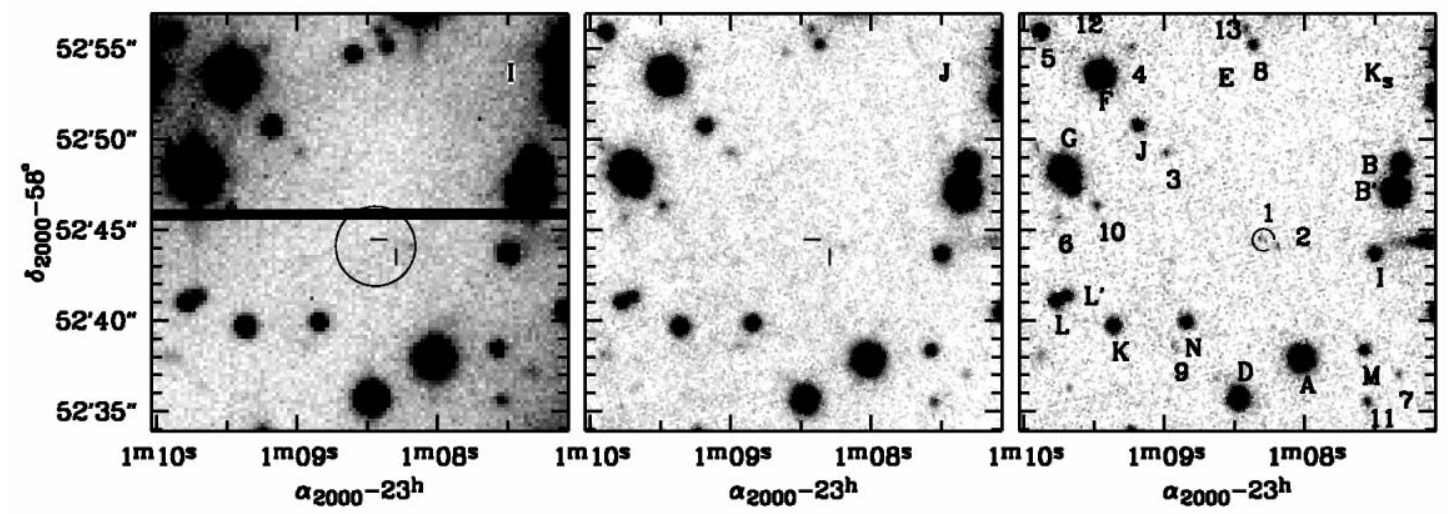

FIG. 1.-Cutouts of the $I$-band image and $K_{s}$ - and $J$-band mosaics of the field around 1E 2259+586. A segment of the Chandra $99 \%$ confidence error circle is overplotted on the $K_{s}$-band image; on all other images the position is indicated by tick marks. The best ROSAT error circle of Hulleman et al. (2000b) is overdrawn on the $I$-band image for reference. We note that although star 1 appears to consist of two dots in $K_{s}$, it is consistent with being a single point source.

the median and standard deviation were computed (excluding physical pixels that were obviously bad). If at any sky position an individual frame differed from the median by more than 3 times the standard deviation, it was discarded and a new standard deviation was computed. This was repeated, until the values of all remaining frames were within 3 standard deviations of the median. The resulting mosaics, shown in Figure 1, have the corresponding mean value at each sky position.

We used DAOPHOT to measure instrumental magnitudes on the mosaics and calibrated these using the infrared standard star SJ 9182 (Persson et al. 1998). In Table 1, we list positions and magnitudes of selected objects (see Fig. 1). We also estimated single-trial $2 \sigma$ detection limits of $23.8 \mathrm{mag}$ in $J$ and 22.4 mag in $K_{s}$ in the way described above.

TABLE 1

Positions and Magnitudes of Selected Objects

\begin{tabular}{|c|c|c|c|c|}
\hline ID & $\begin{array}{c}\alpha \\
(2301+) \\
(\mathrm{J} 2000.0)\end{array}$ & $\begin{array}{c}\delta \\
(+5852+) \\
(\mathrm{J} 2000.0)\end{array}$ & $J$ & $K_{s}$ \\
\hline A & 08.027 & 37.90 & $17.178 \pm 0.012$ & $16.349 \pm 0.006$ \\
\hline & 07.323 & 48.67 & $18.453 \pm 0.012$ & $17.642 \pm 0.009$ \\
\hline & 07.359 & 47.10 & $16.910 \pm 0.011$ & $16.207 \pm 0.008$ \\
\hline & 08.475 & 35.71 & $17.871 \pm 0.012$ & $17.114 \pm 0.007$ \\
\hline $\mathrm{F}$ & 09.454 & 53.51 & $16.609 \pm 0.010$ & $15.908 \pm 0.009$ \\
\hline$G \ldots \ldots$ & 09.721 & 48.26 & $16.449 \pm 0.013$ & $15.957 \pm 0.010$ \\
\hline$H \ldots \ldots$ & 07.079 & 40.51 & $19.763 \pm 0.012$ & $18.963 \pm 0.015$ \\
\hline I . & 07.503 & 43.71 & $20.021 \pm 0.011$ & $19.066 \pm 0.014$ \\
\hline $\mathrm{J}$ & 09.181 & 50.73 & $20.038 \pm 0.013$ & $19.236 \pm 0.018$ \\
\hline $\mathrm{K}$. & 09.361 & 39.71 & $19.497 \pm 0.012$ & $18.651 \pm 0.015$ \\
\hline $\mathrm{L}$ & 09.778 & 41.07 & $20.165 \pm 0.017$ & $19.022 \pm 0.013$ \\
\hline $\mathrm{L}^{\prime}$ & 09.691 & 41.38 & $21.110 \pm 0.034$ & $19.747 \pm 0.028$ \\
\hline M & 07.577 & 38.41 & $20.868 \pm 0.017$ & $19.648 \pm 0.027$ \\
\hline $\mathrm{N}$ & 08.844 & 39.90 & $19.950 \pm 0.012$ & $19.037 \pm 0.014$ \\
\hline $1 \ldots \ldots$ & 08.312 & 44.53 & .. & $21.670 \pm 0.193$ \\
\hline & 08.196 & 44.08 & $23.059 \pm 0.118$ & $21.544 \pm 0.156$ \\
\hline $3 \ldots \ldots$ & 08.985 & 49.23 & $22.299 \pm 0.072$ & $21.079 \pm 0.092$ \\
\hline 4 & 09.226 & 55.04 & $22.378 \pm 0.094$ & $21.094 \pm 0.131$ \\
\hline $5 \ldots \ldots$ & 09.876 & 55.91 & $19.667 \pm 0.011$ & $18.360 \pm 0.012$ \\
\hline 6 & 09.751 & 45.68 & $22.416 \pm 0.079$ & $21.288 \pm 0.124$ \\
\hline 7 & 07.329 & 37.07 & $22.435 \pm 0.075$ & $21.261 \pm 0.084$ \\
\hline 8 & 08.366 & 55.18 & $21.235 \pm 0.025$ & $19.939 \pm 0.023$ \\
\hline 9 & 08.929 & 38.63 & $22.803 \pm 0.111$ & $21.535 \pm 0.186$ \\
\hline $10 \ldots \ldots$ & 09.480 & 46.34 & $21.790 \pm 0.045$ & $20.836 \pm 0.050$ \\
\hline $11 \ldots \ldots$ & 07.552 & 35.55 & $21.824 \pm 0.050$ & $20.651 \pm 0.067$ \\
\hline 12. & 09.643 & 54.90 & $23.322 \pm 0.219$ & $21.956 \pm 0.296$ \\
\hline $13 \ldots \ldots$ & 08.416 & 56.06 & $22.021 \pm 0.053$ & $21.072 \pm 0.084$ \\
\hline
\end{tabular}

Notes. - Units of right ascension are seconds, and units of declination are arcseconds. The errors quoted are the formal fitting errors. The zero-point errors are 0.05 mag in both $J$ and $K_{s}$.

\subsection{Optical Astrometry}

We determined coordinates relative to the USNO-A2.0 catalog (Monet et al. 2000). For the 60 inch images, 1107 USNOA2.0 stars overlap. We determine centroids for 942 objects that were not saturated and appeared stellar, corrected these for instrumental distortion (J. Cohen 1997, private communication), and solved for zero-point position, scale, and position angle. After rejecting 193 outliers, the rms residuals are 0"30 in both right ascension and declination. Therefore, the accuracy of the tie to the USNO-A2.0 system is very good, with a formal uncertainty of 0"01 (standard deviation of the mean). The real uncertainty may be limited by uncertainties in the distortion correction, but we believe it should still be better than 0.03 .

The optical Keck images are too deep and the infrared images cover too small a field for a direct tie to the USNO-A2.0 catalog. Instead, for those, we used stars in common with a short $R$-band exposure, for which Hulleman et al. (2000b) had determined coordinates in the USNO-A2.0 system. For all bands, the tie to the short $R$-band image is accurate to better than $0^{\prime \prime} 02$, and the final tie to the USNO-A2.0 system should again be better than 0.03 .

\section{THE X-RAY POSITION}

1E 2259+586 was observed with Chandra on 2000 January 11, using the Advanced CCD Imaging Spectrometer (ACIS). The analysis of this observation is described in the companion Letter by Patel et al. (2001). Here we use only the data collected in timed exposure mode to derive a position for $1 \mathrm{E} 2259+59.6$. We used the data that were "reprocessed" on 2000 October 31 through the standard pipeline. This reprocessing corrected minor errors in the aspect solution compared to the original processing. Furthermore, in a calibration update made on 2001 July 26, the ACIS pixel size was changed from $0 " 492$ to 0.49131 . We applied this correction manually.

For $1 \mathrm{E} 2259+586$, which is rather bright, the data suffer from pileup of photons in the image core, as a result of which the source looks ring-shaped with a hole in the center. We modeled the data using a Gaussian multiplied by a hyperbolic tangent in radius, scaled to approach zero at $r=0.0$. The resulting best-fit centroids correspond, using the Chandra aspect solution, to a J2000.0 position on the sky of $\alpha=23^{\mathrm{h}} 01^{\mathrm{m}} 08.26, \delta=+58^{\circ} 52^{\prime} 44^{\prime \prime} .9$. The uncertainty is limited by systematic effects, to a circle with $\sim 0$ ".7 radius (Aldcroft et al. 2000).

To improve this position, we tried to identify optical coun- 
terparts to other sources in the field and use these to obtain a boresight correction. For this purpose, we use all seven other sources detected by the source-finding algorithm in the central (S3) chip (see Table 2). Three of the sources (X1, X5, and X6) have X-ray spectra with a soft component, characteristic of stellar sources with little absorption. All of these have counterparts on the Digitized Sky Survey (DSS) plates and positions in the USNO-A2.0 catalog. Object X1 is RP1 of Rho \& Petre (1997); van den Berg \& Verbunt (2001) found a G7-K1 IV-III star with $V=11.8 \pm 0.1$ coincident with RP1. The expected $\mathrm{X}$-ray luminosity of the star agrees with that measured for RP1. The stars coinciding with objects X5 and X6 are relatively bright as well (USNO-A2.0 blue and red magnitudes of 17.5 and 16.6 [X5] and 17.7 and 15.3 [X6]); given the small probability of finding stars this bright at the X-ray position by chance, we conclude that these most likely are the optical counterparts.

Interestingly, no counterparts are seen on the DSS for any of the other sources, which have harder X-ray spectra. However, for X2, which has a low absorbing column, we do find a faint blue star on our Palomar images. Given the unusual color, we believe this is the counterpart. Furthermore, for X7, which appears absorbed, we find a faint extended object at the edge of our $1997 R$ - and $I$-band LRIS images. Given the crowdedness of the field, we cannot be sure this is the counterpart; if it is, $\mathrm{X} 7$ is most likely a highly reddened extragalactic object. Finally, source X4 also overlaps with the 1997 LRIS images but is in a very confused region.

In Table 2, we list positions inferred from our Palomar images, from the USNO-A2.0 catalog, as well as positions from the DSS (both epochs) using astrometric calibrations relative to the USNO-A2.0 catalog. None of the objects appear to have significant proper motion. We used X1 and X6 to establish the boresight correction and X2 to verify our solution. We did not use X5 because it is faint and relatively far off-axis in the ACIS image, and it is not covered by our Palomar data.

In the Chandra images, X1, X2, and X6 are off-axis and thus appear elongated, which may bias the position derived by the source-finding algorithm. For each X-ray source, we computed a model PSF that was rotated by the spacecraft roll angle to match the image orientation and fitted this model to a twodimensional elliptical Gaussian. The offset of the center of this Gaussian from the origin tells us the expected size of the image shift introduced by the Gaussian fit. We then fit a Gaussian with the same $\sigma_{x}, \sigma_{y}$ and inclination to the actual data and subtract the offset determined from the model PSF. We also list the uncertainty in the centroid location estimated from the fit to the actual data.

Armed with both optical and X-ray coordinates for these objects, we adjusted the Chandra coordinate system using the constant offsets, $\delta x$ and $\delta y$, that gave the best agreement with the Palomar positions. The resulting best $\mathbf{J} 2000.0$ position for $1 \mathrm{E} 2259+586$ is $\alpha=23^{\mathrm{h}} 01^{\mathrm{m}} 08.295, \delta=+58^{\circ} 52^{\prime} 44^{\prime \prime} .45$. We stress that this position is tied directly to our optical images, so the only source of systematic uncertainty in the position is the uncertainty in the offset, of $0^{\prime \prime} .19$ in each coordinate. This dominates over the statistical uncertainty and corresponds to a $99 \%$ confidence error radius of 0.60 . The corrected Chandra position is indicated in Figure 1.

\section{IDENTIFICATION OF A COUNTERPART}

We searched for possible counterparts within the Chandra error circle. We did not find any counterpart in our $R, I$, and $J$ images and place upper limits of 26.4, 25.6, and $23.8 \mathrm{mag}$,
TABLE 2

Chandra X-Ray Point Sources on S3 and Their (Tentative) Counterparts

\begin{tabular}{|c|c|c|c|}
\hline Source $^{a}$ & $\begin{array}{c}\alpha \\
(\mathrm{J} 2000.0)\end{array}$ & $\begin{array}{c}\delta \\
(\mathrm{J} 2000.0)\end{array}$ & $\begin{array}{c}\sigma_{\alpha, \delta} \\
(\operatorname{arcsec})\end{array}$ \\
\hline $\mathrm{K} 1\left(1^{\prime \prime} 9,310\right)^{\mathrm{b}}$ & 230033.389 & +585247.12 & 0.09 \\
\hline $1425-14436845^{c}$ & 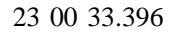 & +585247.16 & 0.2 \\
\hline $\mathrm{DSS}^{\mathrm{d}}$. & 230033.393 & +585247.23 & 0.2 \\
\hline $\mathrm{DSS}^{\mathrm{d}}$ & 230033.380 & +585247.04 & 0.2 \\
\hline $\mathrm{P} 60^{\mathrm{d}}$ & $2300 \quad 33.402$ & +585246.96 & 0.03 \\
\hline $\mathrm{X} 2(1$ & 230043.360 & +585030.44 & 0.06 \\
\hline $\mathrm{P} 60^{\mathrm{d}}$ & 230043.373 & +585030.11 & 0.09 \\
\hline X3 $(1.1,66)$ & 230046.351 & +585308.76 & 0.11 \\
\hline $\mathrm{X} 4(1.2,32)$ & 230055.044 & +585459.05 & 0.25 \\
\hline X5 $(3 " .5,37)$ & 23005 & +585911.03 & 0.72 \\
\hline $1425-14449773^{c}$ & 230059.798 & +585911.68 & 0.2 \\
\hline $\mathrm{DSS}^{\mathrm{d}}{ }^{\mathrm{d}} \ldots \ldots \ldots$ & 230059.816 & $\begin{array}{r}+585911.51 \\
\end{array}$ & 0.2 \\
\hline $\mathrm{DSS}^{\mathrm{d}}$ & 230059.815 & +585911.69 & 0.2 \\
\hline X6 $(2 . .3,380)$ & 230100.118 & +585731.01 & 0.11 \\
\hline $1425-1444992$ & 230100.032 & +585731.12 & 0.2 \\
\hline $\mathrm{DSS}^{\mathrm{d}}$ & 230100.038 & +585731.00 & 0.2 \\
\hline $\mathrm{DSS}^{\mathrm{d}}$ & 230100.107 & +585731.20 & 0.2 \\
\hline $\mathrm{P} 60^{\mathrm{d}}$ & 230100.105 & +585731.17 & 0.03 \\
\hline$X 7^{\mathrm{e}}(0,9,27)$ & 230107.175 & +584941.62 & 0.09 \\
\hline
\end{tabular}

Notes. - Units of right ascension are hours, minutes, and seconds, and units of declination are degrees, arcminutes, and arcseconds. All sources except X5 were on the Palomar images. Except for X1, X2, and X6, no object is found at the position of any of these sources on these images. All X-ray positions were shifted by +0 ".28 in R.A. and -0 "45 in decl. to match the optical positions. The optical positions are on the system defined by the USNO-A2.0 catalog. Hence, they should be on the International Celestial Reference System to $\sim 0$ ".2. Both the DSS1 and the USNO-A2.0 positions are determined from the same plate. See text for details.

${ }^{\text {a }}$ For the X-ray sources in parentheses, we give the approximate image size and number of counts detected.

${ }^{\mathrm{b}} \mathrm{X} 1$ and X2 are RP1 and RP2 of Rho \& Petre 1997, respectively.

${ }^{\mathrm{C}}$ USNO-A2.0 identifier.

${ }^{d}$ Epochs are 1953.830 for DSS1 and USNO-A2.0, 1989.668 for DSS2, and 2000.885 for P60.

${ }^{\mathrm{e}}$ There is an extended source at this position on the edge of a plate taken with LRIS in 1997.

respectively. The $R$ and $I$ limits are nearly 1 mag better than our previously published values (Hulleman et al. 2000b). However, in the new near-infrared $K_{s}$ image, we find a $K_{s}=$ $21.7 \pm 0.2 \mathrm{mag}$ object ( $\operatorname{star} 1$ ) within the Chandra error circle (Fig. 1).

Another object, star 2, while within the ROSAT error circle (see Hulleman et al. 2000b) is offset from the Chandra position by $0.85 \pm 0.25$, where the error includes the uncertainty in the optical position as well as the systematic uncertainty in the X-ray-to-optical transformation. It is inconsistent with being the near-infrared counterpart of $1 \mathrm{E} 2259+586$ at the $99.9 \%$ confidence level.

It is possible that star 1 is an unrelated background object. The density of such faint objects is $0.04 \mathrm{arcsec}^{-2}$. Hence, thanks to the exquisite astrometric precision of Chandra, the probability of chance coincidence is small, less than $3 \%$.

Thus, most likely we have identified the near-infrared counterpart to $1 \mathrm{E} 2259+586$. Patel et al. (2001) report $N_{\mathrm{H}}=$ $9.3 \pm 0.3 \times 10^{21} \mathrm{~cm}^{-2}$ which, using $A_{V}=N_{\mathrm{H}} /\left(1.79 \times 10^{21}\right)$ mag (Predehl \& Schmitt 1995), corresponds to the following extinction values: $A_{R}=4.3, A_{I}=3.1, A_{J}=1.4$, and $A_{K}=$ $0.6 \mathrm{mag}$, which should be accurate to at least a factor of 2 . Adopting these values, we show the optical through X-ray photometry of $1 \mathrm{E} 2259+586$ in Figure 2. Also shown are the blackbody and power-law components of the best-fit model to the X-ray data (Patel et al. 2001).

As for $4 \mathrm{U} 0142+61$, we find an extremely large X-ray- 


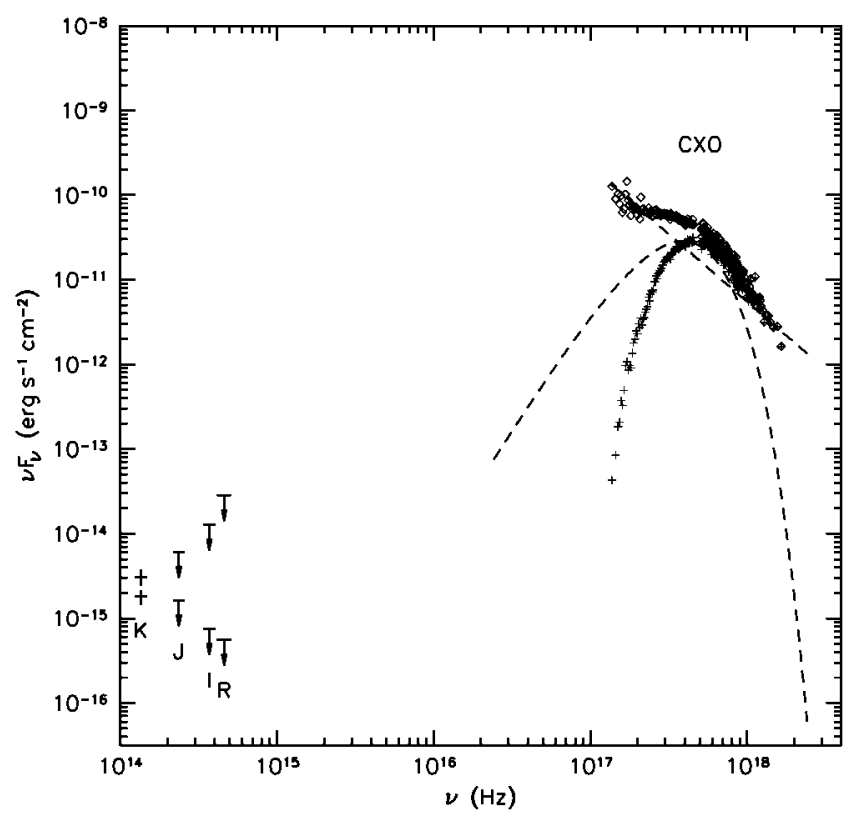

FIG. 2.-Broadband emission spectrum of 1E $2259+586$. Denoted by CXO are the X-ray data (Patel et al. 2001): plus signs are absorbed and diamonds unabsorbed X-ray fluxes. Shown as dashed lines are the blackbody and powerlaw components of the best-fit model. Also shown are the optical and infrared limits and the $K_{s}$-band detection, as observed and after correction for interstellar reddening.

to-optical/infrared flux ratio, which is hard to square with most models proposed for AXPs. Models in which the source is powered by accretion from a fossil disk (e.g., Chatterjee et al. 2000) were already excluded by our previous optical limits. However, this was for a standard disk in which optical emission is due to reprocessing of the X-ray flux.

Hulleman et al. (2000a) showed that for 4U 0142+61, a disk with an inefficiently radiating inner part was excluded as well, but that a truncated disk, such as might be present in a very tight binary, was still possible. For 1E 2259+586, we can also exclude this possibility, since the $K$-band flux and optical limits are inconsistent with the predicted roughly Rayleigh-Jeans spectral energy distribution. For the same reason, we can exclude the possibility that $1 \mathrm{E} 2259+586$ is a hot white dwarf.

A model that may account for the current optical/IR findings, although so far it lacks predictions, is that of Thompson \& Duncan (1996), in which AXPs are magnetars, powered by the decay of a superstrong, $\sim 10^{15} \mathrm{G}$, magnetic field. We hope that our work will stimulate further study into these models. In this respect, we are encouraged by the work done on the X-ray emission by, e.g., Perna et al. (2001) and Özel et al. (2001) and the qualitative agreement between observations and rough estimates of the expected optical flux made by C. Thompson (2001, private communication).

With two counterparts identified, observational progress should be rapid. It should be relatively easy to determine the broadband spectral energy distributions of both sources and identify other AXPs. More difficult, but potentially more rewarding, would be searches for optical pulsations and polarization.

We thank David Kaplan for his help with the near-infrared reductions, for undertaking the LRIS observations, and for donating Palomar observing time. We thank Bryan Jacoby for performing the Palomar observations. The referee (G. L. Israel) is thanked for a careful reading of the manuscript. Part of the observations reported here were obtained at the W. M. Keck Observatory, which is operated by the California Association for Research in Astronomy, a scientific partnership among California Institute of Technology, the University of California, and NASA. It was made possible by the generous financial support of the W. M. Keck Foundation. Part of this work was done while F. H., M. H. v. K., and C. K. were visiting the Institute for Theoretical Physics, which is supported by NSF grant PHY 99-07949. F. H. thanks the Netherlands Organization for Scientific Research and the Leids Kerkhoven Bosscha Fund for grants that made this visit possible. M. H. v. K. acknowledges support from a fellowship of the Royal Netherlands Academy of Science, S. R. K. grants from NSF and NASA, and C. K. and S. K. P. grants NAG5-9591 and GO01018X.

\section{REFERENCES}

Aldcroft, T. L., Karovska, M., Cresitello-Ditmar, M. L., Cameron, R. A., \& Markevitch, M. L. 2000, Proc. SPIE, 4012, 650

Chatterjee, P., Hernquist, L., \& Narayan, R. 2000, ApJ, 534, 373

Hulleman, F., van Kerkwijk, M. H., \& Kulkarni, S. R. 2000a, Nature, 408, 689

Hulleman, F., van Kerkwijk, M. H., Verbunt, F. W. M., \& Kulkarni, S. R. 2000b, A\&A, 358, 605

Israel, G. L., Mereghetti, S., \& Stella, L. 2002, Mem. Soc. Astron. Italiana, in press

Liu, M., \& Graham, J. 2001, ApJ, 557, L31

Matthews, K., \& Soifer, B. T. 1994, in Astronomy with Arrays, The Next Generation (Astrophys. Space Sci. Library 190; Dordrecht: Kluwer), 239

Monet, D., et al. 2000, USNO-A2.0: A Catalog of Astrometric Standards (Flagstaff: USNO)
Oke, J. B., et al. 1995, PASP, 107, 375

Özel, F., Psaltis, D., \& Kaspi, V. M. 2001, ApJ, 563, 255

Patel, S. K., Kouveliotou, C., Woods, P. M., Tennant, A. F., Weisskopf, M. C., Finger, M. H., Göğüş, E., van der Klis, M., \& Belloni, T. 2001, ApJ, 563, 45

Perna, R., Heyl, J. S., Hernquist, L. E., Juett, A. M., \& Chakrabarty, D. 2001, ApJ, 557, 18

Persson, S. E., Murphy, D. C., Krzeminski, W., Roth, M., \& Rieke, M. J. 1998, AJ, 116, 2475

Predehl, P., \& Schmitt, J. H. M. M. 1995, A\&A, 293, 889

Rho, J., \& Petre, R. 1997, ApJ, 484, 828

Thompson, C., \& Duncan, R. C. 1996, ApJ, 473, 322

van den Berg, M., \& Verbunt, F. 2001, A\&A, 368, 569 